\section{Daten unterstreichen: Kein Absetzen der Therapie aus Angst vor SARS-CoV-2}

Weltweit wird daran geforscht, die Auswirkungen einer SARS-CoV-2-Infektion auf Patienten mit entzündlich-rheumatischen Erkrankungen zu erfassen und damit Handlungsempfehlungen für die Therapie von Patienten zu entwickeln. Ob Patienten mit entzündlich-rheumatischen Erkrankungen besonders gefährdet sind, sich mit dem Virus zu infizieren und ob sie - im Falle einer Infektion - ein erhöhtes Risiko haben, einen schweren Verlauf der Viruserkrankung zu entwickeln, ist weitestgehend unbekannt.

Aus vorsichtigem Verantwortungsbewusstsein für ihre Patienten hat die Deutsche Gesellschaft für Rheumatologie e.V. (DGRh) daher bereits im Februar in einer in einer Mitteilung auf ihrer Homepage darauf hingewiesen, dass Patienten mit entzündlichrheumatischen Erkrankungen die Empfehlungen des Robert-Koch-Instituts zur Vermeidung von Kontakten zu anderen Personen und zur Einhaltung von Hygienemaßnahmen wie alle andere Patientengruppen auch strikt befolgen sollten. Gleichzeitig hat die DGRh davon abgeraten, Medikamente, die Patienten mit entzündlich-rheumatischen Erkrankungen zur Behandlung ihrer Erkrankung einnehmen, allein aus Furcht vor einer SARS-CoV-2 Infektion zu pausieren, zu reduzieren oder abzusetzen. Die Sorge ist dabei groß, dass bei Patienten, die ihre Therapie nicht mehr einnehmen, die Rheumaerkrankung wieder aktiv werden könnte und dann zum Abfangen des Erkrankungsschubs sogar höhere Mengen von immunsuppressiven Medikamenten wie Kortison notwendig wären.

Zugleich wurden erste Studien mit Biologika zur Therapie von schweren Verläufen von COVID-19 publik, die nahelegten, dass einige Biologika möglicherweise eine günstige Wirkung für den Fall einer schweren COVID-19 assoziierten Pneumonie haben könnten. Im März hat die DGRh differenzierte Handlungsempfehlungen zum Management von Patienten mit entzündlich- rheumatischen Erkrankungen erstellt und diese ebenfalls auf ihrer Homepage veröffentlicht. Auch hier kommt die DGRh zu der Empfehlung, dass anti-rheumatische Medikamente einer laufenden Therapie nicht ohne Vorliegen einer nachgewiesenen SARS-CoV-2-Infektion abgesetzt werden sollten. Über diese Empfehlungen wurden alle Mitglieder der DGRh im Newsletter informiert, die Zeitschrift für Rheumatologie wird die Empfehlungen demnächst publizieren.

In einer an der Universität Erlangen in Zusammenarbeit der Kliniken für Rheumatologie, Gastroenterologie und Dermatologie erstellten Analyse konnte jetzt gezeigt werden, dass bei signifikant weniger Patienten unter laufender Therapie einer entzündlichen gastroenterologischen, rheumatologischen oder dermatologischen Erkrankung Antikörper gegen SARS-CoV-2 im Serum nachzuweisen waren, als bei 2 Kontrollgruppen - bei Mitarbeitern des Gesundheitssystems und bei einer gesunden, nicht im Gesundheitssystem beschäftigten Bevölkerung. Zudem zeigten die mit Biologika behandelten Patienten im Zeitraum von Februar bis April signifikant seltener Symptome von Atemwegserkrankungen. Die Autoren haben aus diesen Beobachtungen den Schluss gezogen, dass Patienten mit entzündlichen Erkrankungen aus den Bereichen Gastroenterologie, Rheumatologie und Dermatologie, die mit Biologika behandelt wurden, kein erhöhtes Risiko einer Infektion mit SARS-CoV-2 gegenüber den beiden Kontrollgruppen aufwiesen und dass eine Infektion mit dem Virus möglicherweise bei den Patienten weniger schwer verläuft.

Die Autoren unterstützen damit durch ihre Daten die Empfehlungen der DGRh, dass Patienten unter einer laufenden Therapie ihrer entzündlichen rheumatologischen, dermatologischen oder gastroenterologischen Erkrankung keine Risikogruppe per se darstellen und dass sie daher die Therapie allein aus Furcht vor einer Infektion mit SARS-CoV-2 nicht absetzen sollten. Diese Daten sind sehr wichtig für das zukünftige Management von Patienten mit entzündlichen Erkrankungen in der SARS-CoV-2-Pandemie und sie werden auch bei der Aktua- 
lisierung der Empfehlungen der DGRh berücksichtigt werden.

Leider ist bei der verständlichen Freude über die ersten wissenschaftliche Daten zum nicht vorhandenen Risiko unserer Patienten unter einer laufenden Therapie in der Pressemitteilung der Universität Erlangen auch der Hinweis verbreitet worden, Patienten mit entzündlichen Erkrankungen seien durch ihre Therapie vor einer SARSCoV-2-Infektion geschützt. Die DGRh sieht diese Schlussfolgerung als unbegründet und gefährlich. Gemeinsam mit den Autoren der Studie, die sich ausdrücklich von dieser Einschätzung der Pressemitteilung distanzieren, weist die DGRh darauf hin, dass die Daten der Studie zwar beruhigend in Bezug auf die Risiken der SARS-CoV-2-Infektion und auf den Verlauf einer COVID-19 Erkrankung sind, dass sie aber keinesfalls eine Schlussfolgerung dahingehend zulassen, dass Patienten mit entzündlichen rheumatologischen, gastroenterologischen oder dermatologischen Erkrankungen durch ihre Therapie vor einer Infektion oder vor einem potentiell auch tödlichen Verlauf einer COVID-19 Infektion geschützt sind.

Patienten mit entzündlichen rheumatischen, gastroenterologischen und dermatologischen Erkrankungen sollten daher weiterhin konsequent die Hygiene- und $\mathrm{Ab}$ standsempfehlungen des Robert-Koch-Instituts befolgen. Es besteht - darauf weisen die Daten aus Erlangen noch einmal hin keine Notwendigkeit, eine laufende Therapie aus Furcht vor einer SARS-CoV-2-Infektion zu unterbrechen. Aber es gibt auch keinen Grund, in der aktuellen Situation sorglos zu sein oder gar Biologika ohne medizinische Indikation zum Schutz vor einer SARSCoV-2-Infektion oder einem schweren Verlauf einer COVID-19-Erkrankung als Vorbeugung einzunehmen.

Nach einer Pressemitteilung der DGRh 